\title{
Improving ILI Surveillance using Hospital Staff Influenza- like Absence (ILA)
}

\author{
Lydia Drumright ${ }^{* 1}$, Simon D. Frost ${ }^{2}$, Mike Catchpole ${ }^{3}$, John Harrison ${ }^{5}$, Mark Atkins ${ }^{5}$, Penny \\ Parker $^{5}$, Alex J. Elliot ${ }^{4}$, Douglas M. Fleming ${ }^{6}$ and Alison H. Holmes ${ }^{1,5}$

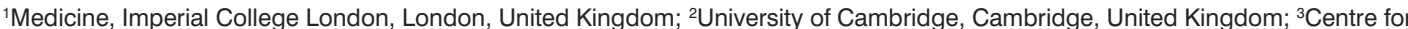 \\ Infections, Health Protection Agency, London, United Kingdom; ${ }^{4}$ Health Protection Agency Syndromic Surveillance Team, \\ Birmingham, United Kingdom; ${ }^{5}$ mperial College Healthcare NHS Trust, London, United Kingdom; ${ }^{6}$ Royal College of General \\ Practitioners Research and Surveillance Centre, Birmingham, United Kingdom
}

\section{Objective}

To address the feasibility and efficiency of a novel syndromic surveillance method, monitoring influenza-like absence (ILA) among hospital staff, to improve national ILI surveillance and inform local hospital preparedness.

\section{Introduction}

Surveillance of influenza in the US, UK and other countries is based primarily on measures of influenza-like illness (ILI), through a combination of syndromic surveillance systems, however, this method may not capture the full spectrum of illness or the total burden of disease. Care seeking behaviour may change due to public beliefs, for example more people in the UK sought care for $\mathrm{pH} 1 \mathrm{~N} 1$ in the summer of 2009 than the winters of 2009/2010 and 2010/2011, resulting in potential inaccurate estimates from ILI (1). There may also be underreporting of or delays in reporting ILI in the community, for example in the UK those with mild illness are less likely to see a GP (2), and visits generally occur two or more days after onset of symptoms (3). Work absences, if the reason is known, could fill these gaps in detection.

\section{Methods}

Weekly counts and rates of hospital staff ILA (attributed to colds or influenza) were compared to GP ILI consultation rates (Royal College of General Practitioners Weekly Returns Service)(4) for 15-64 year olds, and positive influenza A test results (PITR) for all inpatients hospitalised in the three London hospitals for which staff data were collected using both retrospective time series and prospective outbreak detection methods implemented in the surveillance package in $\mathrm{R}(5)$

\section{Results}

Rates of ILA were about six times higher than rates of ILI. Data on hospital staff ILA demonstrated seasonal trends as defined by ILI. Compared to the ILI rates, ILA demonstrated a more realistic estimate of the relative burden of pandemic H1N1 during July 2009 (1) (Figure). ILA provides potentially earlier warnings than GP ILI as indicated by its ability to predict ILI data for the local region ( $\mathrm{p}<$ 0.001 ), as well as its potential for daily 'real time' updates. Using outbreak detection methods and examining peak weeks, alarms and thresholds, ILA alarmed, reached threshold rates and peaked consistently earlier or in the same week as ILI and PITR, with the exception of the July 2009, suggesting that it may be predictive of both community and patient cases of influenza (Table).

\section{Conclusions}

This study has demonstrated the potential to further explore the usefulness of using ILA data to complement existing national influenza surveillance systems. This work could improve our accuracy in monitoring of influenza and has the potential to improve emergency response to influenza for individual hospitals.
Table: Week of the year that alarms commenced and peaks were reached for each of the four official influenza events from March 2008 to April 2011.

\begin{tabular}{|c|c|c|c|c|c|c|c|c|c|c|c|c|}
\hline & \multicolumn{3}{|c|}{ Winter 2008/2009 } & \multirow{2}{*}{\multicolumn{3}{|c|}{ Winter 2009/2010 }} & \multicolumn{3}{|c|}{ Winter 2010/2011 } & \multicolumn{3}{|c|}{ Summer 2009} \\
\hline & Commen & aresh & Peal & & & Peak & ommes & hresh & Peal & omme & hresho & Peak \\
\hline ILA & 33 & 37 & 51 & 38 & 36 & 44 & 47 & 47 & 51 & 27 & 24 & 29 \\
\hline ILI & 36 & 49 & 51 & 3 & 40 & 49 & & 49 & 51 & 26 & 26 & 29 \\
\hline PITR & 49 & $\mathrm{NA}$ & 51 & 39 & NA & 44 & 47 & NA & 52 & 25 & NA & 31 \\
\hline
\end{tabular}

ILA = influenza like absences among hospital staff; ILI = influenza like illness from RCGP data in London, ages 15-64; PITR = positive influenza A test results among patients from the same hospital as staff contributing ILA data; Threshold for ILI data was set at 30/100,000 as defined by the Health Protection Agency. The ILA threshold set at 60/100,000, such that all ILI above a threshold of 30/100000 were also above a threshold for ILA.

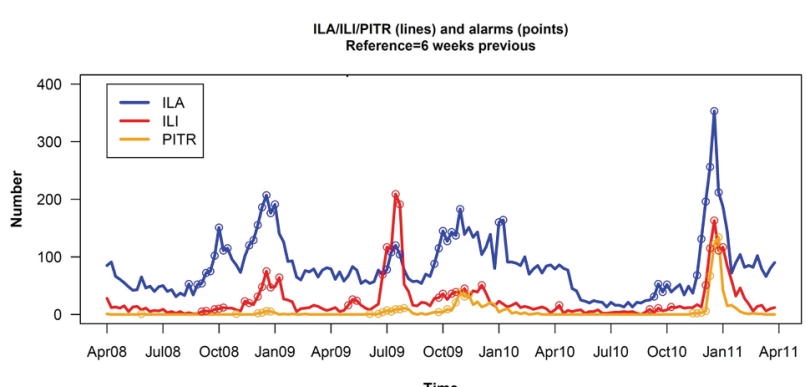

Figure: Weekly counts of ILA among hospital staff (blue), PITR among hospital patients (orange), and ILI in the community (red) from April 2008 to March 2011 and prospective alarms for elevated counts (circles) using a Bayesian subsystem algorithm, using the previous six weeks as the reference for prediction. Data plotted by counts rather than rates for clarity.

\section{Keywords}

influenza; syndromic surveillance; hospital staff; emergency prepardness

\section{References}

1) Birrell PJ, et al. Bayesian modeling to unmask and predict influenza A/H1N1pdm dynamics in London. Proceedings of the National Academy of Sciences 2011;108(45):18238-18243.

2) Evans B, et al. Has estimation of numbers of cases of pandemic influenza H1N1 in England in 2009 provided a useful measure of the occurrence of disease? Influenza and other respiratory viruses 2011.

3) Ross AM, et al. Presentation with influenza-like illness in general practice: implications for use of neuraminidase inhibitors. Communicable Disease and Public Health 2000;3:256-260.

4) Fleming DM. Weekly Returns Service of the Royal College of General Practitioners. Communicable Disease and Public Health 1999;2(2):96-100.

5) Hoehle M. An R package for the monitoring of infectious diseases. Computational Statistics 2007;22(4):571-582.

*Lydia Drumright

E-mail: I.drumright@imperial.ac.uk 